\title{
Metastatic Choroidal Melanoma Presenting as Neovascular Glaucoma
}

\author{
Gregory Fliney $^{\mathrm{a}} \quad$ Osama Ahmed $^{\mathrm{b}} \quad$ Benjamin Young ${ }^{\mathrm{a}} \quad$ Christopher Teng $^{\mathrm{a}}$ \\ Renelle Pointdujour-Lima, c \\ aDepartment of Ophthalmology and Visual Science, Yale University School of Medicine, \\ New Haven, CT, USA; ${ }^{b}$ Yale University School of Medicine, New Haven, CT, USA; ${ }^{\circ}$ Yale \\ Smilow Cancer Center, Yale University School of Medicine, New Haven, CT, USA
}

\section{Keywords}

Neovascular glaucoma $\cdot$ Uveal melanoma $\cdot$ Case report

\begin{abstract}
Uveal melanoma is the most common primary intraocular tumor in adults and can have varying presentations, although it is frequently asymptomatic. One rare presentation of uveal melanoma is neovascular glaucoma (NVG). We present a case of a 20 -year-old male who presented with 2 weeks of left eye redness and decreased vision who was found to have NVG. He was referred for evaluation of glaucoma. Fundoscopic and ultrasonographic examination revealed a mushroom-shaped choroidal mass with low internal reflectivity consistent with choroidal melanoma. The patient underwent enucleation, and metastatic workup revealed hepatic metastases. The patient died 18 months after initial diagnosis. This case emphasizes the importance of a complete ophthalmic evaluation in cases presenting with NVG of unclear etiology to exclude the presence of a potentially life-threatening intraocular tumor.
\end{abstract}

\section{Introduction}

Uveal melanoma, the most common primary intraocular tumor in adults, has an incidence of 5.1 cases per million people in the USA and a median age of diagnosis of 62 years [1, 2]. The presentation of uveal melanoma can vary, and patients are frequently asymptomatic [3]. Early diagnosis of uveal melanoma allows for early treatment which may potentially decrease the risk of metastasis [4]. Once uveal melanoma metastasizes, the prognosis is 
Fig. 1. Slit-lamp photograph of the left eye demonstrates diffuse rubeosis iridis (yellow arrow), a middilated pupil, ectropion uvea, and temporal retinal tissue posterior to the lens (red arrow).

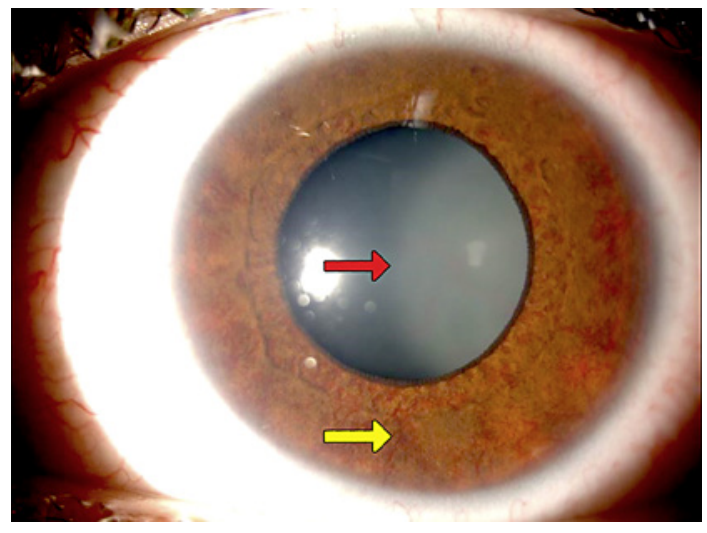

dismal with a median overall survival ranging 9-12.5 months [5, 6]. We present a case of a 20 -year-old male with metastatic choroidal melanoma who initially presented with neovascular glaucoma (NVG).

\section{Case Presentation}

A 20-year-old white male with no past ocular or medical history presented with 2 weeks of redness and decreased vision of the left eye (OS). He was referred for evaluation of NVG. On examination, the visual acuity was $20 / 20$ in the right eye (OD) and no light perception OS. Intraocular pressures were $14 \mathrm{~mm} \mathrm{Hg}$ OD and $53 \mathrm{~mm} \mathrm{Hg}$ OS. Slit-lamp examination showed a normal anterior segment OD and diffuse conjunctival injection, shallow anterior chamber, fixed, mid-dilated pupil, rubeosis iridis, and ectropion uveae OS. An exudative retinal detachment was visualized just posterior to the lens (shown in Fig. 1). Gonioscopic examination revealed an open angle $\mathrm{OD}$ and a narrow angle with peripheral anterior synechiae to the pigmented trabecular meshwork with $360^{\circ}$ of neovascularization of the angle OS. Fundoscopic examination revealed a bullous retinal detachment with no view of the optic disc OS. Anterior segment optical coherence tomography (OCT) showed a flat iris with $360^{\circ}$ of iridotrabecular contact OS. Ultrasonography demonstrated a large, mushroom-shaped choroidal mass with low internal reflectivity $O S$ and retinal detachment. The tumor thickness measured $12 \mathrm{~mm}$ with a $22-\mathrm{mm}$ base and was overhanging the optic disc. There was no evidence of extraocular extension. A clinical diagnosis of choroidal melanoma was established. MRI of the orbits showed a hyperintense intraocular mass with associated subretinal fluid OS and no evidence of extraocular extension of the choroidal melanoma (shown in Fig. 2A).

Given the large choroidal tumor size and poor visual potential, he underwent enucleation OS 3 days after initial presentation. Histopathologic evaluation showed choroidal malignant melanoma with mixed epithelioid and spindle type B cells. There was no evidence of extrascleral extension, and the American Joint Committee on Cancer classification was stage IIIA (shown in Fig. 3). Oncomine genetic analysis showed variants of GNA11 and MYC, and DecisionDx-UM genetic expression assay revealed the tumor to be class 2 .

A metastatic workup with an abdominal MRI revealed a 4.4-cm right hepatic lobe lesion with approximately 10 satellite lesions (shown in Fig. 2B). CT-guided biopsy of the right hepatic lobe confirmed metastatic malignant melanoma. Given that multiple liver lobes had metastases, he was not a candidate for surgical resection with radiotherapy. Treatment of the hepatic metastases included 6 percutaneous hepatic perfusions with intra-arterial melphalan as part of a clinical trial over the course of 9 months without response. He subsequently had 3 treatments 


\section{Case Reports in Ophthalmology}
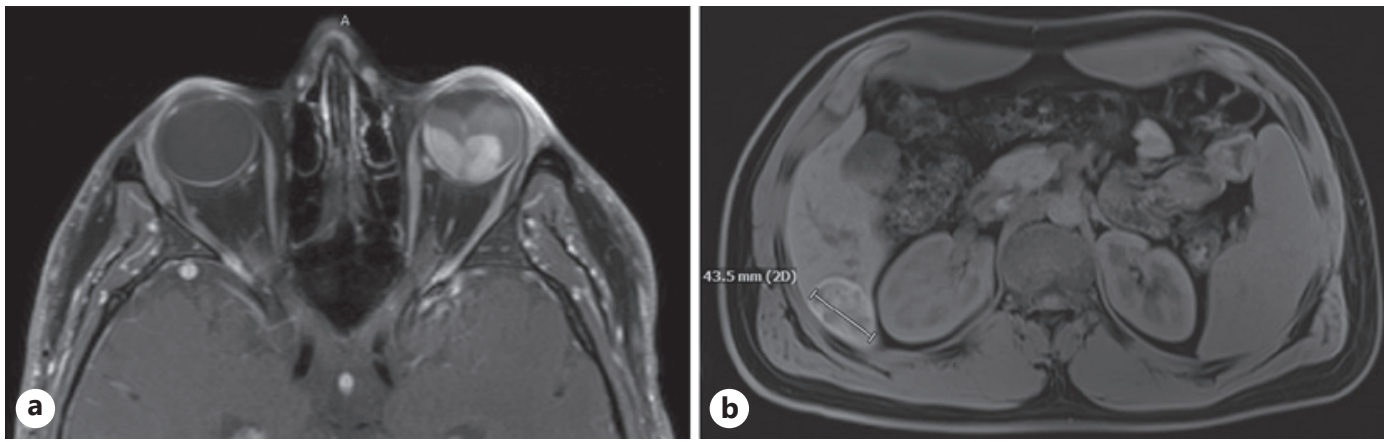

Fig. 2. a T1-weighted MRI of the orbits (axial cut) demonstrates a left intraocular hyperintense mushroomshaped mass without evidence of extraocular extension. Note the overlying, hypointense retinal detachment. b T1-weighted MRI of the abdomen shows a right hepatic lobe hemorrhagic melanoma metastasis.

Fig. 3. H\&E stain of choroidal malignant melanoma showing a heavily pigmented zone predominantly composed of spindle B cells and a minimally pigmented zone composed exclusively of epithelioid cells. There is a high mitotic rate, and the Ki-67 index is approximately $15 \%$.

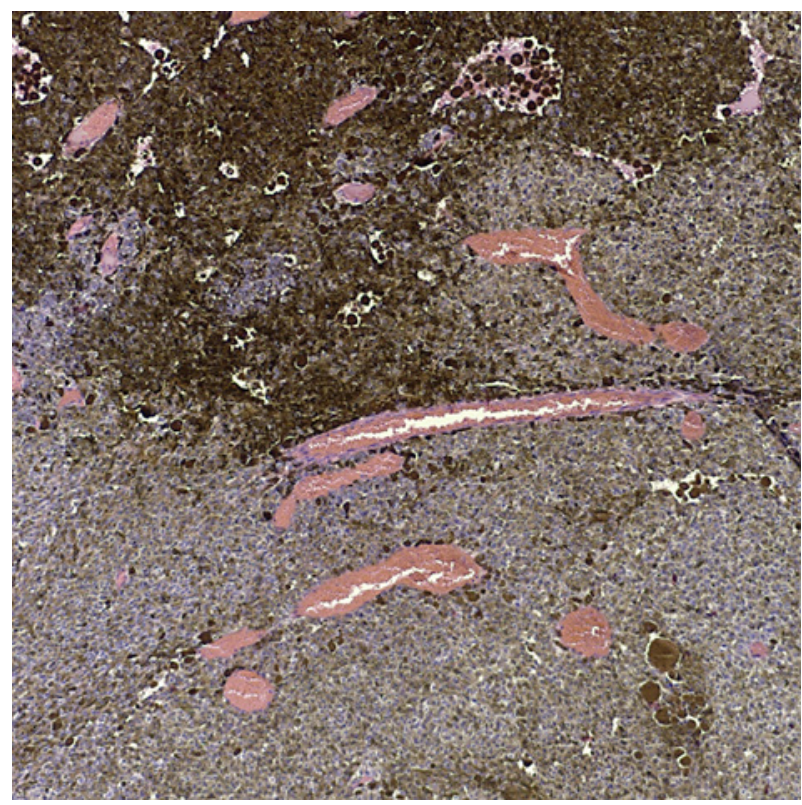

of intra-arterial hepatic immunoembolization. Following these treatments, abdominal MRI revealed new splenic and vertebral lesions with an increase in the number of hepatic lesions concerning for worsening metastasis. He received 2 cycles of immunotherapy, ipilimumab and nivolumab, with concurrent granulocyte-macrophage colony-stimulating factor.

He ultimately developed immune therapy-related hepatitis and was found to have hepatic vein thrombi. He was started on heparin and developed a hemorrhage of the small intestine that could not be safely intervened upon. Despite attempts at transfusion, he died from hemorrhagic shock 18 months after his initial diagnosis.

\section{Discussion}

Uveal melanoma accounts for $3.1 \%$ of reported melanoma cases and has an annual incidence of 5.1 cases per million in the USA [1]. The median age of diagnosis is 62 years, and only $1.5 \%$ of cases present in patients under 21 years of age [2]. Although metastasis 
portends a dismal outcome in uveal melanoma, it is present in $<4 \%$ of cases at the time of presentation in the general population [7]. Of note, patients under 21 years of age have a $9.4 \%$ reduced 10 -year risk of metastasis compared to the general population [3]. In light of these figures, we present an exceptionally uncommon case of a 20 -year-old patient who presented with NVG secondary to a large choroidal melanoma with metastases to the liver.

Shields et al. [8] found that choroidal melanoma induces elevated intraocular pressure in $2 \%$ of cases, most of which are due to NVG. The mechanism of NVG is driven by the expression of proangiogenic factors such as vascular endothelial growth factor and fibroblast growth factor by the ischemic and necrotic tumor. Although NVG is much more commonly caused by retinal ischemic conditions such as retinal venous obstructive disease (36\%), diabetic retinopathy (32\%), or carotid artery obstructive disease (13\%), it may be the only apparent sign of an otherwise insidious choroidal melanoma [9].

The patient described did not have any documented symptoms and was unaware of his loss of vision. Uveal melanoma has an asymptomatic presentation in $30 \%$ of cases, resulting in a delayed diagnosis [10]. Dilated fundus examination and ancillary testing including OCT, fundus autofluorescence, and ultrasonography are the mainstay of making the clinical diagnosis. Dilated fundus examination often reveals a pigmented $(92 \%)$ or amelanotic $(8 \%)$ choroidal mass with characteristic features including subretinal fluid, orange pigmentation, proximity to the optic disc (within $3 \mathrm{~mm}$ ), and the absence of drusen [11]. OCT is used to confirm the presence of subretinal fluid. Fundus autofluorescence is used to confirm the presence of lipofuscin or orange pigment. Ultrasonography demonstrates a dome- or mushroom-shaped mass with $>2 \mathrm{~mm}$ of thickness and low internal reflectivity. These characteristic features can help distinguish melanoma from similar appearing "pseudomelanomas," such as choroidal nevus, exudative hemorrhagic chorioretinopathy, and congenital hypertrophy of the retinal pigment epithelium. Fine-needle aspiration biopsy can be helpful in making the diagnosis for indeterminant tumors.

Uveal melanoma metastasizes to the liver in $93 \%$ of metastatic cases [12]. Abdominal imaging using CT or MRI is routinely used, with MRI emerging as the superior option for minimizing radiation exposure and providing increased sensitivity for small tumors [3]. To date, no study has demonstrated improved survival with early detection of metastatic disease [3].

Treatment of uveal melanoma is highly individualized and varies by tumor size. Our patient presented with a tumor thickness of $12 \mathrm{~mm}$, and he underwent enucleation followed by liver-directed experimental therapy and systemic immunotherapy. Small progressive and medium-sized tumors (3-10 $\mathrm{mm}$ ) can be treated with plaque brachytherapy or proton beam radiation. Large tumors $(>10 \mathrm{~mm})$, or those not amenable to treatment by radiation, are treated with enucleation $[3,13]$. Although no systemic adjuvant therapy for metastatic uveal melanoma has improved the median overall survival of 9-12.5 months thus far, novel treatments such as targeted therapy and immunotherapy are currently being trialed $[3,5,6]$. Immunotherapy has had disappointing initial results for the treatment of metastatic uveal melanoma with a study of ipilimumab reporting an overall survival of 6.8 months from the time of metastasis [14]. Our patient received a combination of ipilimumab and nivolumab, which is currently being studied in 2 phase II clinical trials [13].

Prognosis in uveal melanoma depends mostly on its potential to metastasize. Shields et al. [15] have described a strong positive correlation between the thickness of the primary melanoma and its metastatic potential. Other clinical risk factors for metastasis include extraocular growth and involvement of the ciliary body. Age younger than 21 years tends to have a more favorable prognosis with a $9 \%$ metastasis rate at 10 years from the time of

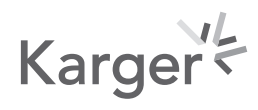


diagnosis compared to $25 \%$ at all ages $[2,13]$. Prognostic data rely on tumor DNA or RNA. Cytogenetic changes to chromosomes 3 and $8 \mathrm{q}$ correlated strongly with a poor prognosis, whereas chromosome 6 abnormalities portend a better prognosis [3]. Somatic tumor gene expression profiling is RNA based, with mutations in genes such as BAP1 portending a worse prognosis, and mutations in EIF1AX and SF3B1 genes being associated with late metastasis and better prognoses [3]. These genes, in addition to a panel of others, are used to classify uveal melanoma class 1A (best prognosis), 1B (intermediate), or 2 (worst prognosis). Gene expression profiling of the tumor in our patient revealed class 2 using DecisionDx-UM, which is associated with a younger presentation, larger tumor height, and decreased time to metastasis [16].

Our patient's clinical course emphasizes the potentially insidious nature of choroidal melanoma and the devastating outcomes of metastasis. Neovascularization as the presenting sign of uveal melanoma is rare. However, it is important to consider uveal melanoma in the setting of neovascularization of unclear origin.

\section{Statement of Ethics}

The photographs of the patient were taken after obtaining written informed consent from the patient for publication of this case report and any accompanying images. The provisions of the Declaration of Helsinki for research involving human subjects were followed. This study protocol was reviewed, and the need for approval was waived by the Yale Institutional Review Board.

\section{Conflict of Interest Statement}

The authors have no conflicts of interest to declare.

\section{Funding Sources}

There were no funding sources.

\section{Author Contributions}

Gregory Fliney and Osama Ahmed wrote the manuscript. Osama Ahmed was also a medical student on the patient's Medical Oncology inpatient team. Benjamin Young was an ophthalmology resident participating in the patient's care and edited the manuscript. Christopher Teng was the primary glaucoma specialist managing the patient at the time of presentation and edited the manuscript. Renelle Pointdujour-Lim was the primary ophthalmic oncologist managing the patient since presentation. She also contributed to the editing of the manuscript.

\section{Data Availability Statement}

All data generated or analyzed during this study are included in this article.

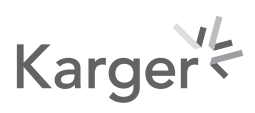




\section{References}

1 Singh AD, Turell ME, Topham AK. Uveal melanoma: trends in incidence, treatment, and survival. Ophthalmology. 2011 Sep;118(9):1881-5.

2 Shields CL, Kaliki S, Arepalli S, Atalay HT, Manjandavida FP, Pieretti G, et al. Uveal melanoma in children and teenagers. Saudi J Ophthalmol. 2013 Jul;27(3):197-201.

3 Chattopahdyay C, Kim DW, Gombos D, Oba J, Qin Y, Williams M, et al. Uveal melanoma: from diagnosis to treatment and the science in between. Cancer. 2016 Aug;122(15):2299-312.

4 Straatsma BR, Diener-West M, Caldwell R, Engstrom RE, Collaborative Ocular Melanoma Study Group. Mortality after deferral of treatment or no treatment for choroidal melanoma. Indian J Ophthalmol. 2018 Oct; 66(10):1395-400.

5 Rietschel P, Panageas KS, Hanlon C, Patel A, Abramson DH, Chapman PB. Variates of survival in metastatic uveal melanoma. J Clin Oncol. 2005 Nov;23(31):8076-80.

6 Kath R, Hayungs J, Bornfeld N, Sauerwein W, Höffken K, Seeber S. Prognosis and treatment of disseminated uveal melanoma. Cancer. 1993 Oct; 72(7):2219-23.

7 Finger PT, Kurli M, Reddy S, Tena LB, Pavlick AC. Whole body PET/CT for initial staging of choroidal melanoma. Br J Ophthalmol. 2005 Oct;89(10):1270-4.

8 Shields CL, Shields JA, Shields MB, Augsburger JJ. Prevalence and mechanisms of secondary intraocular pressure elevation in eyes with intraocular tumors. Ophthalmology. 1987 Jul;94(7):839-46.

9 Brown GC, Magargal LE, Schachat A, Shah H. Neovascular glaucoma: etiologic considerations. Ophthalmology. 1984 Apr;91(4):315-20.

10 Kaliki S, Shields CL. Uveal melanoma: relatively rare but deadly cancer. Eye. 2017 Feb;31(2):241-57.

11 Blasi MA, Laguardia M, Tagliaferri L, Scupola A, Villano A, Caputo CG, et al. Brachytherapy alone or with neoadjuvant photodynamic therapy for amelanotic choroidal melanoma: functional outcomes and local tumor control. Retina. 2016 Nov;36(11):2205-12.

12 Collaborative Ocular Melanoma Study Group. Assessment of metastatic disease status at death in 435 patients with large choroidal melanoma in the Collaborative Ocular Melanoma Study (COMS): COMS report no. 15. Arch Ophthalmol. 2001 May;119(5):670-6.

13 Yang J, Manson DK, Marr BP, Carvajal RD. Treatment of uveal melanoma: where are we now? Ther Adv Med Oncol. 2018 Feb;10:1758834018757175.

14 Zimmer L, Vaubel J, Mohr P, Hauschild A, Utikal J, Simon J, et al. Phase II DeCOG-study of ipilimumab in pretreated and treatment-naïve patients with metastatic uveal melanoma. PloS One. 2015;10(3):e0118564.

15 Shields CL, Furuta M, Thangappan A, Nagori S, Mashayekhi A, Lally DR, et al. Metastasis of uveal melanoma millimeter-by-millimeter in 8033 consecutive eyes. Arch Ophthalmol. 2009 Aug;127(8):989-98.

16 Berry D, Seider M, Stinnett S, Mruthyunjaya P, Schefler AC, Ocular Oncology Study Consortium. Relationship of clinical features and baseline tumor size with gene expression profile status in uveal melanoma: a multiinstitutional study. Retina. 2019;39(6):1154-64. 